\section{Interferencia en la absorción de levotiroxina asociada al uso de orlistat}

$\mathrm{U}$

na mujer de 45 años con antecedentes de hipotiroidismo primario autoinmune y obesidad consulta por primera vez al servicio de endocrinología por síntomas compatibles con hipotiroidismo a pesar de encontrarse en tratamiento con levotiroxina (Tirogal ${ }^{\circledR}$ ) $200 \mu \mathrm{g}$ al día. Se cambia la marca del medicamento a Eutirox ${ }^{\circledR}$ conservando la dosis y la paciente acude nuevamente a consulta 5 meses después con síntomas de tirotoxicosis (figura 1); en esta consulta se decide iniciar orlistat $120 \mathrm{mg}$ tres veces al día como tratamiento para su obesidad, así mismo se disminuye la dosis de levotiroxina. Diez meses después refiere nuevamente síntomas de hipotiroidismo y se encuentra nuevamente TSH elevada, para esta fecha la dosis de levotiroxina había sido incrementada por su médico de atención primaria. Se decide suspender el orlistat sospechando una interferencia en la absorción inducida por el medicamento y disminuir la dosis de levotiroxina a la cantidad usual que tomaba la paciente. Seis semanas después la paciente se encontraba nuevamente con una TSH baja y requirió un nuevo

Figura 1. Variación de la TSH de acuerdo a la administración de orlistat

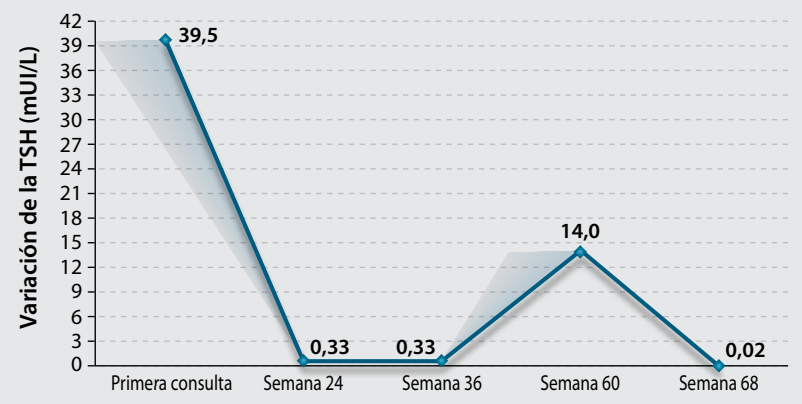

ajuste de la dosis. Posteriormente fue sometida a cirugía bariátrica (sleeve gástrico) para el manejo de su obesidad.

El orlistat interfiere con la absorción de múltiples medicamentos ${ }^{(1)}$ pero su interacción con la levotiroxina es escasamente reportada. El primer paciente descrito en la literatura presentó síntomas de hipotiroidismo e incremento en la TSH sérica dos semanas después de iniciar el medicamento y sus síntomas y niveles de TSH mejoraron 2 semanas después de la supresión del medicamento ${ }^{(2)}$. El segundo caso reportado en la literatura mostró como una paciente en tratamiento con orlistat requirió incrementos graduales en la dosis de levotiroxina con el uso del medicamento y al suspenderlo nuevamente requirió disminución en la dosis.

Debido a los pocos casos reportados la forma de interacción no es muy clara, se propone que el orlistat puede unirse a la levotiroxina limitando su absorción o que éste sea sólo un efecto de la esteatorrea y malabsorción producida por el orlistat o que la disminución en la absorción de sales de yodo pudieran causar un hipotiroidismo y esto se tradujera en la necesidad de incrementar la dosis de levotiroxina, no se han documentado vías metabólicas comunes en la cuales pudiera existir interferencia de dichos medicamentos. Por el momento no existe ninguna recomendación de no administrar ambos medicamentos de manera concomitante, la sugerencia es que estos se administren con 4 horas de diferencia.

\section{Carlos Esteban Builes Montaño}

Hospital Pablo Tobón Uribe, Universidad de Antioquia, Medellín, Colombia.

Carlos Alfonso Builes Barrera

Hospital San Vicente Fundación, Universidad de Antioquia, Medellín, Colombia

\section{Referencias}

1. Liwanpo L, Hershman JM. Conditions and drugs interfering with thyroxine absorption. Best Pract Res Clin Endocrinol Metab. diciembre de 2009;23(6):781-92.

2. Madhava K, Hartley A. Hypothyroidism in thyroid carcinoma follow-up: orlistat may inhibit the absorption of thyroxine. Clin Oncol (R Coll Radiol). septiembre de 2005;17(6):492. 